\title{
Prolonged Survival for Choroid Plexus Carcinoma with Oncocytic Changes: A Case Report
}

Melissa Ann Eppinger*, Casey Melissa Berman, Steven L. Halpern and Catherine Anne Mazzola

Department of Neurosciences, Goryeb Children's Hospital - Morristown Medical Center, Morristown, New Jersey

\begin{abstract}
Introduction: Choroid plexus carcinoma (CPC) is a rare central nervous system neoplasm derived from the choroid plexus epithelium. CPCs are challenging to surgically resect due to their intraventricular location and high vascularity [1]. Tumor recurrence and dissemination are frequently seen with $\mathrm{CPC}$. Due to the malignant nature of these tumors, the prognosis is poor with a five year survival rate of approximately $40 \%$ [2].

Case Description: The authors report a pediatric patient with a CPC (WHO grade III) with oncocytic changes, who survived for seven years after her initial diagnosis. A 14-month-old female presented to the emergency department with a sudden onset change in mental status following a fall. Imaging revealed a hemorrhagic, left intraventricular heterogeneous tumor. The patient underwent a left parietal craniotomy and gross tumor resection. Pathological findings were consistent with a malignant CPC. Her treatment regimen included four cycles of etoposide, Temodar, Cytoxan and vincristine, two cycles of oral etoposide and temozolomide with vincristine, in addition to a stem cell harvest. Recurrence of our patient's disease led to two subsequent gross total resections. This patient was in the minority who survived greater than five years with this tumor type.

Conclusion: Gross total resection is the goal, and pharmacologic intervention including chemotherapy and radiation therapy remains controversial, as this treatment is not curative [3]. We would like to share this unusual case with the medical community to increase clinical understanding in the management and treatment of this malignant tumor.
\end{abstract}

Keywords: Choroid plexus tumors; Choroid plexus carcinoma; CPC; Oncocytic

\section{Manuscript}

Choroid plexus tumors (CPTs) are rare, intraventricular neoplasms that originate from the epithelial cells of the choroid plexus [1-4]. CPTs account for $0.4 \%$ to $0.6 \%$ of all central nervous system (CNS) tumors $[1,2,5,6]$. The incidence of CPTs are slightly higher within the pediatric population, as they represent $1.5 \%-3.9 \%$ of all CNS tumors in this age group [5]. CPTs include both benign and malignant variants. The majority of CPTs are benign choroid plexus papillomas, which exhibit excellent prognosis [7]. Choroid plexus carcinomas (CPC) are rare, malignant neoplasms that represent $20-30 \%$ of CPTs in children [5]. CPC is primarily a pediatric malignancy, as $80 \%$ of them occur in the pediatric population [2]. The median age for CPC development is 26 to 32 months old $[2,6]$. The World Health Organization (WHO) has classified a CPC as a WHO grade III, and prognosis is poor with a five year survival rate of approximately $40 \%[2,8]$. Histologic features include necrotic and cystic regions, distortion of the papillary pattern, increased mitotic figures, and nuclear pleomorphism $[1,2,9]$. Two-thirds of CPCs spread into the cerebrospinal fluid (CSF) and disseminate throughout this pathway [5]. In children, CPCs are most commonly found in the lateral ventricles, in contrast to adults who most frequently develop these tumors in the fourth ventricle [10]. This malignancy typically presents with signs of hydrocephalus and increased intracranial pressure [7]. Although the exact mechanism is unclear, this is believed to be due to overproduction of CSF by the tumor cells, blockage of CSF flow, or impaired CSF absorption $[7,8]$.

We report an unusual case of a pediatric patient with a choroid plexus carcinoma (WHO grade III) with oncocytic changes, who survived for seven years.

\section{Case Report}

A 14-month-old female presented to the emergency department with a sudden onset change in mental status following a fall from a couch. Several weeks prior to the accident, it was noted that the patient had mild macrocephaly and was losing developmental milestones including her ability to walk. In the emergency department, it was observed that the patient was lethargic, had right hemiparesis, as well as a sluggish and slightly dilated left pupil. An emergent computed tomographic (CT) scan revealed a $10 \mathrm{~cm} \mathrm{x} 8 \mathrm{~cm}$ hemorrhagic tumor arising from the left lateral ventricle. After the CT scan, the child became comatose and required intubation while in the emergency department. A Magnetic Resonance Imaging (MRI) scan of the brain was ordered. The MRI revealed a large, hemorrhagic, left intraventricular heterogeneous tumor with multiple stages of bleeding (Figures 1A-1D)

The patient was promptly taken to the operating room, and an emergent left parietal craniotomy and gross total resection (GTR) was performed. A post-operative MRI was done the day after surgery (Figures 2). Figure 2 demonstrates a clean surgical bed with no evidence of residual tumor. Pathological findings were consistent

*Corresponding author: Melissa Ann Eppinger, Department of Neurosciences, Goryeb Children's Hospital - Morristown Medical Center, Morristown, New Jersey, US, Tel: 973-326-9000; Fax: 973-326-9001; E-mail: meppinger@drew.edu

Received January 29, 2016; Accepted March 03, 2016; Published March 10 2016

Citation: Eppinger MA, Berman CM, Halpern SL, Mazzola CA (2016) Prolonged Survival for Choroid Plexus Carcinoma with Oncocytic Changes: A Case Report. J Pediatr Neurol Med 1: 107. doi:10.4172/2472-100X.1000107

Copyright: (c) 2016 Eppinger MA, et al. This is an open-access article distributed under the terms of the Creative Commons Attribution License, which permits unrestricted use, distribution, and reproduction in any medium, provided the original author and source are credited. 

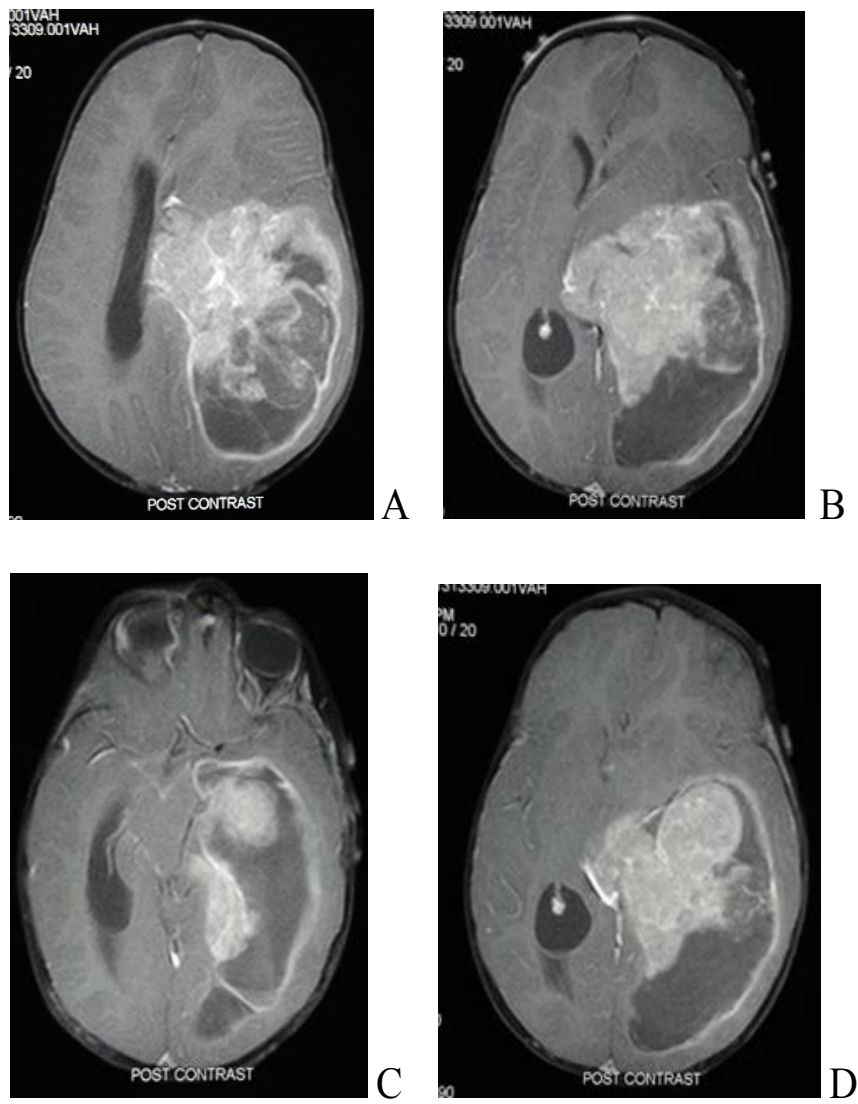

Figure 1: Pre-operative MRI brain with IV contrast demonstrating the left lateral ventricular choroid plexus carcinoma (A) T1W1 axial view 1, (B) T1W1 axial view 2, (C) T1W1 axial view 3, (D) T1W1 axial view 4.
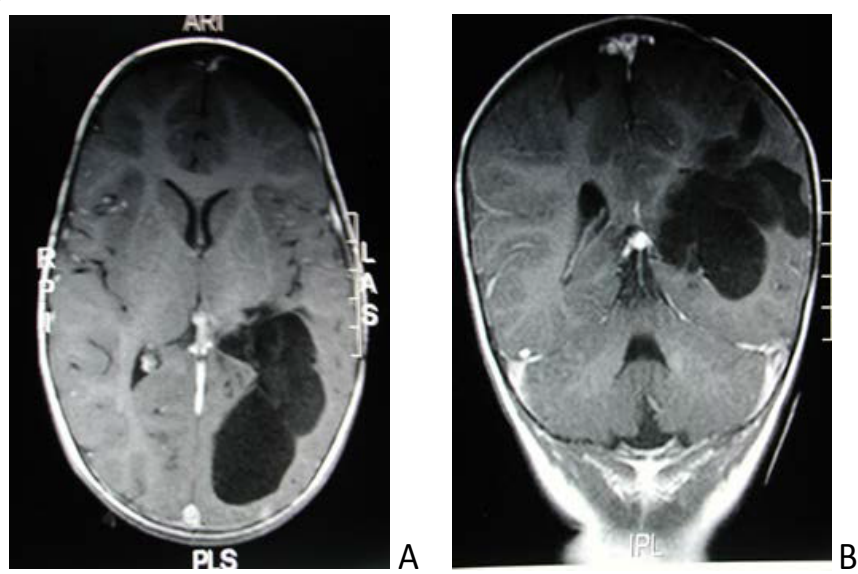

Figure 2: Post-operative MRI brain with IV contrast after tumor resection (A) T1W1 axial view; (B) T1W1 coronal view.

with a malignant CPC displaying both papillary and solid elements. Initial cytology of the CSF came back positive, but was subsequently confirmed to be negative.

After her incision healed, she was cleared for chemotherapy two weeks after surgery. The patient started chemotherapy at 14 months of age. Her treatment regimen consisted of four cycles of etoposide (VP16), Temodar, Cytoxan and vincristine, and two cycles of oral VP-16 and temozolomide with vincristine. Her MRI one month post-operatively revealed a second mass in the left cerebellopontine angle (CPA), which was most likely metastatic tumor from the ventricle. No other lesions were observed in the patient's MRI of the spine. At this time, the patient had lost several developmental milestones.

At 15 months of age, the patient underwent high dose chemotherapy with an autologous stem cell harvest to help the patient recover from treatment. At her two month follow-up appointment, the patient reportedly received physical therapy once a week. The patient was catching up with her developmental milestones.

At 27 months of age, the patient had a follow-up MRI of the brain and spine. A recurrent tumor in the posterior surgical bed measuring approximately $1.21 \mathrm{~cm}$ by $1.02 \mathrm{~cm}$ was reported in the atrium of the left ventricle (Figure 3). Additionally, minimal enhancement was noted near the conus at L1 on her spine films. The left CPA enhancement was unchanged. At this time, the neurosurgical team recommended an emergent craniotomy and resection of recurrent tumor within the posterior parietal resection cavity. The patient underwent a GTR and did well post-operatively. She tolerated the last round of Temodar and VP-16 without complication.

At three years of age, she underwent craniospinal radiotherapy with high energy photons through a collimator involving 3600 cGy at 180 cGy fractions, in addition to a coned down field modification boost of 1800 cGy to 1980 cGy.

The patient was in remission for approximately three and a half years following the completion of all therapeutic interventions. At this time, a follow up MRI of the patient's brain revealed findings compatible with metastatic tumor progression. A new heterogeneously enhancing mass measuring $3.6 \mathrm{~cm}$ x $3.1 \mathrm{~cm}$ transaxially and $3.8 \mathrm{~cm}$ craniocaudally was observed subadjacent to the patient's prior left parietal craniotomy. In addition, findings included enlargement of subadjacent foci of gliosis and encephalomalacia communicating with the ventricular system suggesting CSF tumor spread (Figure 4). Imaging also revealed two lesions in the liver. The patient was six years and three months old with reoccurrence of her $\mathrm{CPT}$.

Palliative care was offered to the family and was being considered.

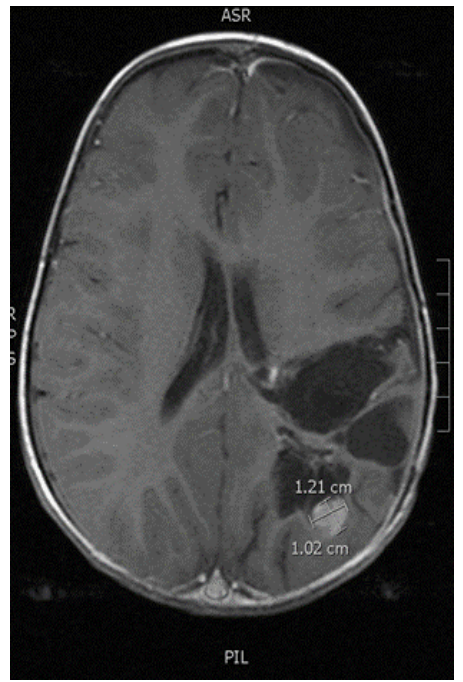

Figure 3: Follow-up MRI scan with IV contrast demonstrating recurrent tumor: T1W1 axial view. 


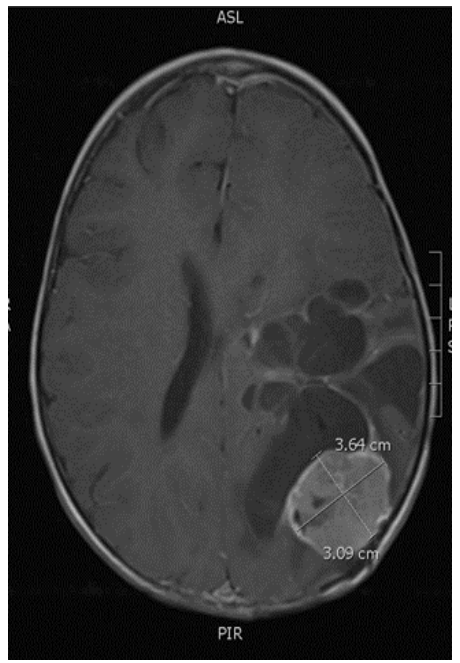

Figure 4: Follow-up MRI brain with IV contrast showing heterogeneous mass, three and a half years after therapy: TWA1 axial view.
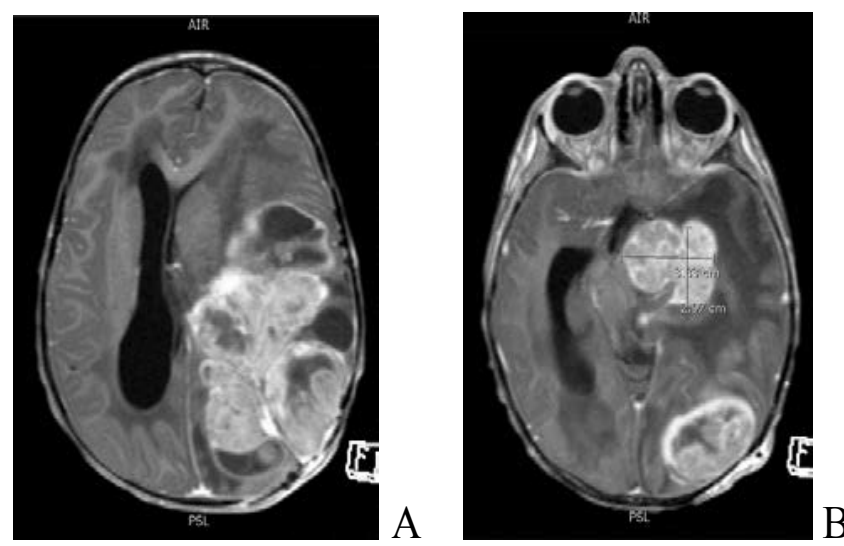

Figure 5: Follow-up MRI brain with IV contrast showing recurrent tumor (A) T1W1 axial view 1, (B) T1W1 axial view 2.

However, approximately two weeks later, the patient presented to the emergency department with signs and symptoms of right-sided weakness and increased intracranial pressure. She was admitted through the emergency department, and a decision was made to pursue a third tumor resection although the surgery would most likely not be curative. The patient underwent a left parietal craniotomy and GTR of the recurrent tumor. No intraoperative complications were reported. Pathology revealed a recurrent CPC diagnosed at WHO grade IV. At this time, chemotherapy was re-administered.

Unfortunately, approximately one year later recurrent tumor was noted on follow-up MRI with contrast (Figures 5). Hospice care was recommended. The patient was now seven years and nine months old. Months after palliative care commenced the child passed away at home with her family present.

\section{Discussion}

$\mathrm{CPC}$ is a rare, malignant neoplasm derived from choroid plexus epithelium. CPCs are challenging to surgically resect due to their intraventricular position and high vascularity [1]. Many of these tumors are large at initial presentation. Tumor recurrence and dissemination are frequently seen with CPC due to the difficulty of complete resection and the malignant nature of these tumors [1]. As we observed with our patient, the high vascularity of this tumor places patients at an increased risk for excessive intra-operative blood loss, which led to the need for several blood transfusions in our case. Intra-operative blood loss is a major cause of morbidity and mortality in pediatric patients [1].

At the present time, there is no established protocol for the treatment of CPCs [2]. GTR is the primary goal, however this is accomplished in only $40-50 \%$ of cases [2]. Several studies compare the results of total versus incomplete resection, establishing GTR as the best possible treatment if possible. In a study of patients with CPCs by Bettegowda et al. $80 \%$ of patients who received GTR remained in remission. However, only $17 \%$ of subjects who received incomplete resection remained in remission, with $83 \%$ experiencing relapse [7]. Wrede et al. conducted a study of 347 patients with CPC, also comparing those who had underwent a complete resection versus subtotal resection [11]. The two year survival rate was $72.9 \%$ for patients with complete resection, and $41.9 \%$ for patients with partial resection. In the case of subtotal resection, a second surgery is recommended [11]. In this same study, $22.6 \%$ of subjects with residual tumor received a subsequent surgery. The two year survival rate for these patients increased to $69 \%$, compared to a two year survival rate of $30 \%$ in those who did not have a second surgery [11].

Therapeutic intervention remains controversial and should be considered on a case by case basis $[1,2,12,13]$. Despite multiple reports about the benefits of chemotherapy and radiation therapy, medical treatment for this tumor type is not curative [3]. In a study of an eight month old infant who achieved remission with chemotherapy, local tumor recurrence was reported 13 months after initiation of treatment [14]. CPCs are aggressive tumors and have a high incidence of recurring and spreading to multiple regions of the body [1]. Several articles recommend chemotherapy before tumor resection in order to increase the probability of complete removal by shrinking the tumor in size and vascularity $[1,3,12,15,16]$ Souweidane et al. reports a patient whose tumor size shrank by $29.5 \%$ after receiving three cycles of chemotherapy consisting of VP-16, cyclophosphamide, vincristine, and cisplatin [16]. However although total surgical resection was achieved, the patient reportedly was only in remission for 31 months [16]. In an article by St. Clair et al. pre-operative chemotherapy including VP-16, ifosfamide, and carboplatinum allowed for a safer two-stage tumor removal, due to reduction of tumor vascularity [3].

It is believed that radiation alone, chemotherapy alone, or combined therapy following subtotal resection can increase survival rate, although this remains controversial [7]. Radiation therapy alone was not suggested for CPCs in the pediatric patient population due to the intolerable and undesirable side effects $[1,15,17]$. Several articles support the use of post-operative treatments such as combined therapies or adjuvant chemotherapy alone $[12,15,17,18]$. In a case report written by Arico et al. a three month old infant went into remission after receiving chemotherapy and delayed irradiation following a non-complete tumor removal [12]. The patient was still in remission 8 years later with only small psychomotor and endocrine deficits [12]. In a study of patients with CPC by Berger et al. 11 subjects who had undergone incomplete resection received post-operative chemotherapy. Four of the 11 patients experienced a complete response with chemotherapy [19]. A systematic literature review was performed by Sun et al. to determine the overall survival rate of patients who received post-operative monothereapy compared to combined treatment [13]. The study concluded that combined therapy versus chemotherapy alone yielded increased 
survival [13]. However, radiation therapy alone was found not to contribute to survival [17]. Due to this tumor's tendency to disseminate throughout the cerebral spinal fluid space, it is believed that treatment of the entire neuraxis is necessary [14].

Although total resection provides the best prognosis, recurrence is also seen in these cases. In Bettegowda et al. study of patients with CPCs who underwent complete resection, four of seven subjects had recurrence [7]. In Berger et al. study, eight subjects received complete CPC resection followed by chemotherapy. Even with this adjuvant therapy, $13 \%$ of subjects experienced tumor recurrence [19]. These studies reflect what we observed with our patient. Although complete resection may provide remission for a period of time, recurrence is common. Further research studies may help clinicians discover the best management and treatment plans for these rare, malignant tumors. We as a medical community would like to spread awareness about CPCs in the hope to reduce morbidity and mortality associated with this disease, in addition to improving patients' quality of life and overall survival [20].

\section{Conclusion}

$\mathrm{CPC}$ is an aggressive tumor, and prognosis is poor. GTR is the goal, and although pharmacologic treatment remains controversial, studies have demonstrated its benefit as adjuvant therapy. Multiple surgical resections along with chemotherapy were able to prolong our patient's life for seven years from initial diagnosis. However despite four cycles of VP-16, Temodar, Cytoxan and vincristine, two cycles of oral etoposide and temozolomide with vincristine, our patient's disease returned about four years after the completion of therapy. Remarkably, this pediatric patient was in the minority who survived longer than five years with a CPC (WHO grade III), which has a five year survival rate of only $40 \%$ [2]. We would like to share this unusual case with the medical community to increase clinical understanding in the management and treatment of this rare, malignant tumor.

\section{References}

1. Ogiwara H, Dipatri AJ Jr, Alden TD, Bowman RM, Tomita T (2012) Choroid plexus tumors in pediatric patients. Br J Neurosurg 26: 32-37.

2. Gopal P, Parker JR, Debski R, Parker JC Jr (2008) Choroid plexus carcinoma. Arch Pathol Lab Med 132: 1350-1354.

3. St Clair SK, Humphreys RP, Pillay PK, Hoffman HJ, Blaser SI, et al. (1991) Current management of choroid plexus carcinoma in children. Pediatr Neurosurg 17: 225-233.

4. Yan C, Xu Y, Feng J, Sun C, Zhang G, et al. (2013) Choroid plexus tumours: classification, MR imaging findings and pathological correlation. J Med Imaging Radiat Oncol 57: 176-183.
5. Kang HS, Wang KC, Kim YM, Kim IO, Kim SK, et al. (1997) Choroid plexus carcinoma in an infant. J Korean Med Sci 12: 162-167.

6. Paulus W, Brandner S (2007) Choroid plexus tumours. In: Louis DN, Ohgaki H Wiestler OD, Cavenee WK, eds. WHO Classification of Tumours of the Central Nervous System. IARC Press, Lyons pp: 81-85.

7. Bettegowda C, Adogwa O, Mehta V, Chaichana KL, Weingart J, et al. (2012) Treatment of choroid plexus tumors: a 20 -year single institutional experience. J Neurosurg Pediatr 10: 398-405.

8. Yip CM, Tseng HH, Hsu SS (2014) Choroid plexus carcinoma: A rare tumor in adult. Surg Sci 5: 146-149.

9. Greenberg MS (2010) Tumor: 21.2.22. In: Choroid plexus tumors. Handbook of neurosurgery. Thieme Medical Publishers, New York pp: 695-696.

10. Mishra A, Ojha BK, Chandra A, Singh SK, Chandra N, et al. (2014) Choroid plexus papilloma of posterior third ventricle: A case report and review of literature. Asian J Neurosurg 9: 238

11. Wrede B, Liu P, Ater J, Wolff JE (2005) Second surgery and the prognosis of choroid plexus carcinoma--results of a meta-analysis of individual cases. Anticancer Res 25: 4429-4433.

12. Aricò M, Raiteri E, Bossi G, Giordana MT, Corbella F, et al. (1994) Choroid plexus carcinoma: report of one case with favourable response to treatment. Med Pediatr Oncol 22: 274-278.

13. Sun MZ, Ivan ME, Oh MC, Delance AR, Clark AJ, et al. (2014) Effects of adjuvant chemotherapy and radiation on overall survival in children with choroid plexus carcinoma. J Neurooncol 2: 353-360.

14. Griffin BR, Stewart GR, Berger MS, Geyer JR, O'Dell M, et al. (1988) Choroid plexus carcinoma of the fourth ventricle. Report of a case in an infant. Pediatr Neurosci 14: 134-139.

15. Duffner PK, Kun LE, Burger PC, Horowitz ME, Cohen ME, et al. (1995) Postoperative chemotherapy and delayed radiation in infants and very young children with choroid plexus carcinomas. The Pediatric Oncology Group. Pediatr Neurosurg 22: 189-196.

16. Souweidane MM, Johnson JH, Lis E (1999) Volumetric reduction of a choroid plexus carcinoma using preoperative chemotherapy. J Neurooncol 43: 167171

17. Pierga JY, Kalifa C, Terrier-Lacombe MJ, Habrand JL, Lemerle J (1993) Carcinoma of the choroid plexus: a pediatric experience. Pediatr Oncol 21: 480-487.

18. Greenberg ML (1999) Chemotherapy of choroid plexus carcinoma. Nerv Syst 15: 571-577.

19. Berger C, Thiesse P, Lellouch-Tubiana A, Kalifa C, Pierre-Kahn A, et al. (1998) Choroid Plexus Carcinomas in Childhood: Clinical Features and Prognostic Factors. Neurosurgery 3: 470-475.

20. Zaky W, Dhall G, Khatua S, Brown RJ, Ginn KF, et al. (2015) Choroid plexus carcinoma in children: The Head Start experience. Pediatr Blood Cancer 5: 784-789. 